\title{
Trajectories of childhood BMI and adult diabetes: the Bogalusa Heart Study
}

\author{
Tao Zhang ${ }^{1} \cdot$ Jie Xu ${ }^{2,3} \cdot$ Shengxu $\mathrm{Li}^{4} \cdot$ Lydia A. Bazzano $^{5} \cdot$ Jiang $\mathrm{He}^{5} \cdot$ Paul K. Whelton $^{5} \cdot$ Wei Chen $^{5}$ (D)
}

Received: 2 August 2018 / Accepted: 24 September 2018 / Published online: 20 October 2018

(C) Springer-Verlag GmbH Germany, part of Springer Nature 2018

\begin{abstract}
Aims/hypothesis The aim of this study was to characterise longitudinal profiles of BMI from childhood and to examine the impact of level-independent childhood BMI trajectories on adult type 2 diabetes.

Methods The longitudinal cohort consisted of 2449 adults (1613 white and 836 black) who had their BMI measured between four and 15 times from childhood (4-19 years) to adulthood (20-51 years) and fasting glucose measured in adulthood. Modelestimated levels and linear slopes of BMI at childhood age points were calculated in 1-year intervals using growth-curve parameters and their first derivatives, respectively.

Results BMI from childhood to adulthood fit cubic growth curves; linear and non-linear curve parameters differed significantly between race-sex groups. BMI showed race and sex differences from 15 years onwards. Individuals with hyperglycaemia had higher long-term BMI levels than those who were normoglycaemic in race-sex groups. Linear and non-linear slope parameters of BMI differed consistently and significantly between adult hyperglycaemia groups. The OR of childhood BMI levels for ages 4 19 years was $1.45-1.83$ ( $p<0.001$ for all) for adult hyperglycaemia after adjustment for confounders. Level-adjusted linear slopes of BMI at ages 10-19 years showed significantly positive associations with adult hyperglycaemia (OR 1.17-1.50, $p<0.01$ for all). The associations of childhood BMI linear slopes with adult hyperglycaemia were not significant during the age period 59 years. The trends in these associations were consistent across race-sex groups.

Conclusions/interpretation These observations indicate that childhood BMI trajectories have a significant impact on adult diabetes, independent of BMI levels. The adolescence age period is a crucial window for the development of diabetes in later life, which has implications for early-life prevention.

Data availability All data and materials are publicly available at the National Heart, Lung, and Blood Institute (NHLBI) Biologic Specimen and Data Repository and can be accessed at https://biolincc.nhlbi.nih.gov/studies/bhs.
\end{abstract}

Keywords Body mass index $\cdot$ Childhood $\cdot$ Diabetes $\cdot$ Hyperglycaemia $\cdot$ Longitudinal study

\section{Abbreviation}

AIC Akaike's information criterion

Tao Zhang and Jie Xu contributed equally to this study.

Electronic supplementary material The online version of this article (https://doi.org/10.1007/s00125-018-4753-5) contains peer-reviewed but unedited supplementary material, which is available to authorised users.

Wei Chen

wchen012@hotmail.com

1 Department of Biostatistics, School of Public Health, Shandong University, Jinan, China

2 Department of Neurology, Beijing Tiantan Hospital, Capital Medical University, Beijing, China
3 China National Clinical Research Center for Neurological Diseases, Beijing, China

4 Cardiovascular Programs, Children's Hospitals and Clinics of Minnesota, Minneapolis, MN, USA

5 Department of Epidemiology, School of Public Health and Tropical Medicine, Tulane University, 1440 Canal Street, New Orleans, LA 70112, USA 


\section{Research in context}

\section{What is already known about this subject?}

- Elevated BMI levels early in life are a risk factor for adult diabetes

What is the key question?

- Is the increasing slope of BMI during childhood predictive of adult diabetes risk?

What are the new findings?

- Linear and non-linear slope parameters of BMI differed significantly between adult hyperglycaemia groups

- Level-independent BMI slopes predicted adult diabetes risk, particularly at adolescence

How might this impact on clinical practice in the foreseeable future?

- The adolescence age period is a crucial window for the development of diabetes in later life, which has implications for early-life prevention

\section{Introduction}

Type 2 diabetes has reached epidemic proportions in the USA. One in three Americans will have diabetes by the year 2050. Clinical and epidemiologic studies have demonstrated an important role of BMI in the development of diabetes [1-4]. Previous studies, including studies from the Bogalusa Heart Study, have established that elevated BMI levels in early life are a risk factor for adult diabetes [5-9]. It is well documented that high levels of childhood BMI are associated with increased glucose levels in both children and adults $[10,11]$. The concept of 'childhood origins' of diabetes is supported by numerous publications from large-scale population-based cohorts in which individuals have been followed since childhood [12]. In addition to the absolute level of BMI, evidence is increasingly accumulating that longitudinal BMI growth trajectories during particular periods of life also have an adverse impact on the development of diabetes [13-16].

Despite a large body of literature that depicts the childhood BMI-adult diabetes relationship, there are substantial gaps in our knowledge regarding the association between childhood BMI trajectory patterns and adult diabetes risk. BMI levels at different ages in childhood represent the obesity status at a particular timepoint, and the rate of increase in BMI in a specific childhood age window reflects the velocity of weight change. A steeper increasing slope of BMI at a particular age point is indicative of the potential for weight gain at a later stage of life. We hypothesised that an increasing slope of BMI during childhood would be predictive of adult diabetes risk, independent of the absolute BMI value at the same timepoint. To test this hypothesis, we required data from a longitudinal cohort, such as the Bogalusa Heart Study, where participants have been followed from early childhood to adulthood.

By using repeated measurements of BMI from childhood to adulthood in the Bogalusa Heart Study, we aimed to characterise longitudinal BMI trajectory profiles from early school age to midlife, and to examine the association of level-independent trajectories of BMI during childhood with adult diabetes risk.

\section{Methods}

All data and materials are publicly available at the NHLBI Biologic Specimen and Data Repository and can be accessed at https://biolincc.nhlbi.nih.gov/studies/bhs.

Study cohort The Bogalusa Heart Study, which has conducted a series of long-term observations in a semi-rural community of mixed races (65\% white and 35\% black) in Bogalusa, Louisiana, USA, was founded by Dr. Gerald Berenson in 1973. It focuses on understanding the early natural history of cardiovascular disease and childhood risk factors [17]. In the community of Bogalusa, nine surveys of children aged 4 19 years and 11 surveys of adults aged 20-51 years who were previously examined as children were conducted between 1973 and 2010. Linking these repeated cross-sectional examinations conducted every $2-3$ years provides serial observations from childhood to adulthood in the same individuals. After excluding participants with non-fasting blood samples and missing data on BMI, glucose, other diagnostic information of type 2 diabetes or covariates, 2449 adult study participants (1613 white and 836 black; $44.3 \%$ male, mean age 34.8 years) formed the longitudinal cohort for this study. These participants had been examined four to 15 times for BMI (7.0 times on average; at least twice in childhood and at least twice in adulthood) and had data on fasting glucose, self-reported type 2 diabetes history and medications.

At each examination, written informed consent was obtained from the study participants or a parent/guardian for those 
under 18 years of age. Study protocols were approved by the institutional review board of the Tulane University Health Sciences Center.

\section{General examinations}

All measurements were obtained by trained study staff members who followed a standard protocol [17]. Participants were instructed to fast for at least $12 \mathrm{~h}$, with compliance ascertained by interview on the morning of screening, and antecubital venous blood was collected to obtain plasma. Weight in light clothing without shoes was measured to the nearest $0.1 \mathrm{~kg}$ on a dedicated scale that was routinely calibrated; height was measured to the nearest $0.1 \mathrm{~cm}$ using a free-standing stadiometer. Height and weight were measured twice, and the mean value was used to calculate BMI as weight in kilograms divided by the square of height in meters. Information on smoking and alcohol use was obtained by means of a staffadministered standardised questionnaire. Current smoking and drinking were defined as smoking at least one cigarette per day and consuming alcohol every day, respectively, during the prior 12 months.

Laboratory analyses Plasma glucose levels were measured as part of a multiple chemistry profile (SMA20; Laboratory Corporation of America, Burlington, NC, USA) using a glucose oxidase method. On the basis of ADA criteria, participants were defined as having type 2 diabetes if they had a fasting plasma blood glucose level of $\geq 7.0 \mathrm{mmol} / 1$ or were taking glucose-lowering medication in adulthood; prediabetes was defined as a fasting plasma glucose level of 5.6$6.9 \mathrm{mmol} / 1$ [18]. The hyperglycaemia group included individuals with prediabetes and type 2 diabetes.

Statistical methods Non-linear growth-curve parameters of BMI measured multiple times from childhood to adulthood were estimated using a random-effects mixed model, as previously reported $[19,20]$. The mixed model incorporates fixed and random effects, and allows the intercept and linear and non-linear parameters to vary from individual to individual. The random-effect coefficients represent the difference between the fixed-effect parameters and the observed values for each individual. This model allows for repeated measurements and different numbers of unequally spaced observations across individuals. The mixed linear model computes maximum likelihood estimates of curve parameters, generating 2449 different sets of curve parameters (i.e. one for each study participant). The model selection was based on Akaike's information criterion (AIC). The most parsimonious model was selected based on $p$ values of the independent variable (age) at a significance level of 0.05 . Age and its higher-order terms were included one by one for model building. The higher- order terms of age were not included in the model if they were not significant or made lower-order terms not significant, or did not improve the goodness of fit of the model based on AIC values. Cubic curves were fitted for BMI in race-sex groups.

$$
\begin{aligned}
\text { BMI }_{\mathrm{i}}= & \left(\beta_{0}+\mathrm{b}_{0 \mathrm{i}}\right)+\left(\beta_{1}+\mathrm{b}_{1 \mathrm{i}}\right) \text { age }+\left(\beta_{2}+\mathrm{b}_{2 \mathrm{i}}\right) \text { age }^{2} \\
& +\left(\beta_{3}+\mathrm{b}_{3 \mathrm{i}}\right) \text { age }^{3}+\varepsilon
\end{aligned}
$$

where $\beta=\left(\beta_{0}, \beta_{1}, \beta_{2}, \beta_{3}\right)$ is a vector of fixed-effect parameters, $b=\left(b_{0}, b_{1}, b_{2}, b_{3}\right)$ is a vector of random-effect parameters and $\varepsilon$ is an unknown error term for the individual i. Age was centred to the mean (20.1 years) to remove the collinearity of age with its higher-order terms. The term age $^{2}$ was divided by 10 and age $^{3}$ by 20 to improve the model fitting.

Electronic supplementary material (ESM) Fig. 1 shows the BMI growth curve of a white male participant as an example to describe the calculation of model-estimated levels and linear slopes of BMI in childhood. The levels and linear slopes (tangent lines) of childhood BMI during 4-19 years of age were calculated using each individual's curve parameters and their first derivatives, respectively. Prior to logistic regression analyses, childhood linear slope values of BMI at each age point were adjusted for their corresponding levels by regression residual analyses, then standardised with $Z$ transformation (mean 0 , SD 1) by race-sex groups to avoid collinearity of levels and linear slopes in the same model.

The significance of differences in mean levels of study variables and curve parameters between race-sex groups was tested using ANCOVA in a generalised linear model. The derived curve parameters of BMI, including levels and linear slopes at childhood ages, were used as predictor variables for association analyses with hyperglycaemia, type 2 diabetes and prediabetes in multivariable logistic regression models, adjusting for sex, race, adult age, and adult smoking and alcohol use. The age periods of 4-11 and 12-19 years were grouped as pre-adolescence and adolescence, respectively.

\section{Results}

Table 1 summarises the study variables by age periods in race-sex groups. Sex differences in BMI were seen from adolescence to adulthood in black (male $<$ female) and white (male $>$ female) participants. Race differences in BMI were significant from adolescence to adulthood in females only (black $>$ white participants). There were 304 hyperglycaemic adults, including $164(6.7 \%)$ with prediabetes and 140 $(5.7 \%)$ with diabetes. The prevalence of prediabetes was higher in males than in females among white participants, while the prevalence of diabetes did not differ significantly between race or sex groups. 
Table 1 Characteristics of study participants by race and sex

\begin{tabular}{|c|c|c|c|c|c|c|}
\hline \multirow[t]{2}{*}{ Age period } & \multicolumn{2}{|l|}{ White } & \multicolumn{2}{|l|}{ Black } & \multicolumn{2}{|c|}{$\begin{array}{l}p \text { for race } \\
\text { difference }\end{array}$} \\
\hline & Male & Female & Male & Female & Male & Female \\
\hline Childhood (4-11 years) & $n=507$ & $n=627$ & $n=261$ & $n=360$ & & \\
\hline Age (years) & $8.0 \pm 2.0$ & $7.9 \pm 2.0$ & $8.1 \pm 2.1$ & $7.9 \pm 2.0$ & 0.848 & 0.982 \\
\hline BMI $\left(\mathrm{kg} / \mathrm{m}^{2}\right)$ & $16.5 \pm 2.4$ & $16.6 \pm 3.0$ & $16.6 \pm 2.5$ & $16.5 \pm 2.5$ & 0.904 & 0.550 \\
\hline Adolescence (12-19 years) & $n=691$ & $n=837$ & $n=353$ & $n=468$ & & \\
\hline Age (years) & $16.7 \pm 2.0$ & $16.7 \pm 2.0$ & $16.9 \pm 1.8$ & $16.9 \pm 1.9$ & 0.056 & 0.039 \\
\hline BMI $\left(\mathrm{kg} / \mathrm{m}^{2}\right)$ & $22.3 \pm 4.4 * *$ & $21.6 \pm 4.4$ & $22.4 \pm 4.8^{* *}$ & $23.5 \pm 5.3$ & 0.619 & $<0.001$ \\
\hline Adulthood (20-51 years) & $n=726$ & $n=887$ & $n=358$ & $n=478$ & & \\
\hline Age (years) & $35.7 \pm 8.8$ & $35.4 \pm 8.9$ & $33.3 \pm 9.5$ & $33.5 \pm 9.1$ & $<0.001$ & $<0.001$ \\
\hline BMI $\left(\mathrm{kg} / \mathrm{m}^{2}\right)$ & $28.7 \pm 6.2 * *$ & $27.7 \pm 7.5$ & $28.1 \pm 7.2 * *$ & $30.6 \pm 8.8$ & 0.146 & $<0.001$ \\
\hline Smokers, $n(\%)$ & $231(31.8)$ & $281(31.7)$ & $136(38.0)^{* *}$ & $115(24.1)$ & 0.051 & 0.004 \\
\hline Alcohol drinkers, $n(\%)$ & $230(31.7)^{* *}$ & $150(16.9)$ & $157(43.9)^{* *}$ & $84(17.6)$ & $<0.001$ & 0.815 \\
\hline Prediabetes, $n(\%)$ & $71(9.8) * *$ & $38(4.3)$ & $27(7.5)$ & $28(5.9)$ & 0.321 & 0.202 \\
\hline Type 2 diabetes, $n(\%)$ & $37(5.1)$ & $42(4.7)$ & $26(7.3)$ & $35(7.3)$ & 0.230 & 0.053 \\
\hline Hyperglycaemia, $n(\%)$ & $108(14.9)^{* *}$ & $80(9.0)$ & $53(14.8)$ & $63(13.2)$ & 0.196 & 0.053 \\
\hline
\end{tabular}

Data are means $\pm \mathrm{SD}$, unless otherwise indicated

$* * p<0.01$ for a sex difference within racial groups
Figure 1 presents the longitudinal trajectories of BMI from childhood to adulthood for 2449 individuals by race and sex. Growth curves in white male and black male participants had similar trajectories. However, BMI increased faster in black female participants than in the other race-sex groups, and this difference was discernible at around age 10 years. White female participants tended to have a lower BMI than the other race-sex groups after age 15 years. Detailed information on curve parameters are presented in ESM Table 1. All curve parameters were significant $(p<0.001)$ except the cubic terms in black participants ( $p=0.353$ for males and $p=0.054$ for females).

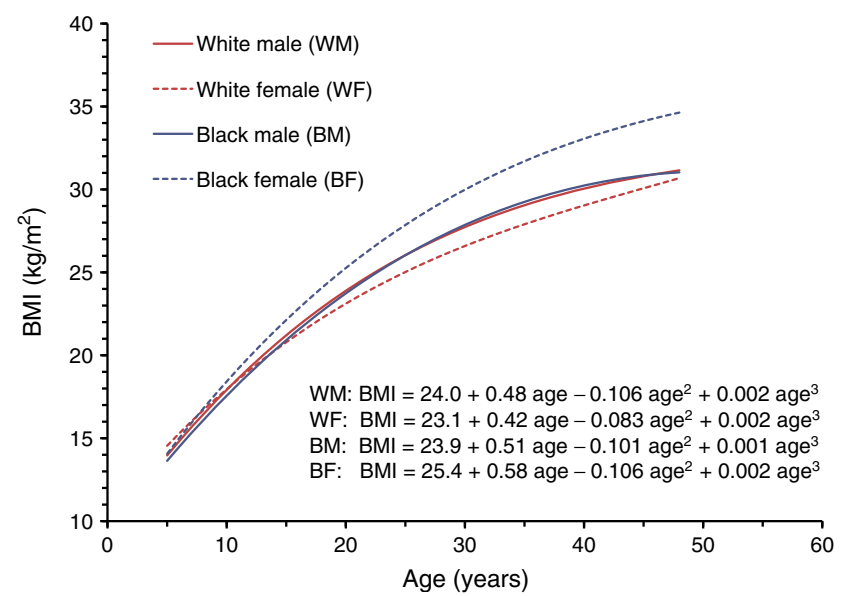

Fig. 1 Growth curves of BMI by race-sex groups, with curve parameters shown for each group. Curve parameters were all significantly different from $0(p<0.001)$ except the cubic terms $0.001(p=0.353)$ in black male participants and $0.002(p=0.054)$ in black female participants
Figure 2 presents the longitudinal trajectory patterns of BMI in participants with hyperglycaemia and normoglycaemia by race and sex. Compared with the normoglycaemic group, hyperglycaemic participants had higher BMI levels $\left(\beta_{0}+b_{0}\right)$ at age 20.1 years and higher linear slopes $\left(\beta_{1}+b_{1}\right.$, tangent lines) at age 20.1 years, but lower non-linear slopes $\left(\beta_{2}+b_{2}\right.$ and $\beta_{3}+b_{3}$ ) from childhood to adulthood. The growth curves of BMI of the two groups started to separate from around age 10 years consistently in the four race-sex groups, with white females having the greatest difference.

Table 2 presents a comparison of curve parameters between hyperglycaemic and normoglycaemic adults by race and sex. The curve parameters $\beta_{0}+b_{0}$ and $\beta_{1}+b_{1}$ of BMI at age 20.1 years were all significantly greater in hyperglycaemic than in normoglycaemic adults; however, the curve parameters $\beta_{2}+b_{2}$ around 20-35 years of age did not differ significantly between the two groups. The curve parameters $\beta_{3}+b_{3}$ around 36-51 years of age were significantly smaller in hyperglycaemia than in normoglycaemia among white females and black males.

ESM Tables 2 and 3 show model-estimated levels and linear slopes of BMI, respectively, during childhood (4-19 years) in 1 -year intervals by race-sex groups. The linear slopes of BMI at childhood ages were calculated for each individual based on the first derivatives of their curve parameters. The linear slopes of BMI in ESM Table 3 were all significantly positive and decreased as age increased. White males had significantly higher values of BMI slopes than white females, but the direction of the sex difference was opposite in black participants. Race differences (black > white participants) in slopes were 
Fig. 2 Growth curves of BMI by race-sex-hyperglycaemia groups: (a) white male; (b) white female; (c) black male; (d) black female. The key applies to all figure parts a
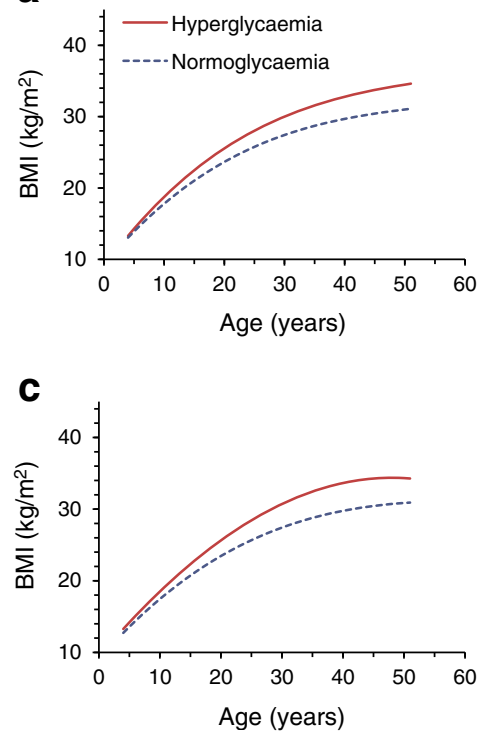

b

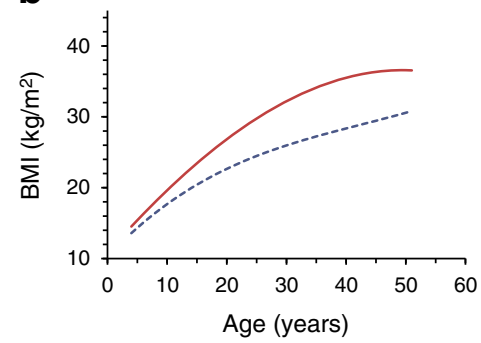

d

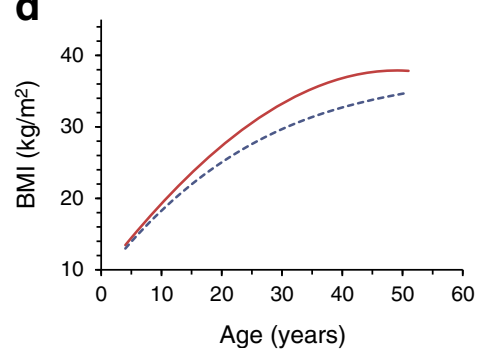

significant for all ages in females. Model-estimated levels and slopes of BMI were significantly and positively correlated at all childhood ages $(p<0.001)$. The correlation patterns were consistent in race-sex groups, with slight changes in correlation coefficient sizes (data not shown).
Figure 3 shows standardised ORs and 95\% CIs of modelestimated BMI levels and level-adjusted BMI linear slopes for adult hyperglycaemia (prediabetes and type 2 diabetes, $n=304$ ) by childhood age, adjusted for adult age, sex, race and adult smoking and alcohol drinking. The OR values of

Table 2 Difference in BMI curve parameters between hyperglycaemic and normoglycaemic groups by race and sex

\begin{tabular}{|c|c|c|c|c|c|c|}
\hline \multirow[t]{2}{*}{ Curve parameter } & \multicolumn{2}{|l|}{ White $(n=1613)$} & \multicolumn{2}{|l|}{ Black $(n=836)$} & \multicolumn{2}{|c|}{$p$ for race difference } \\
\hline & Male & Female & Male & Female & Male & Female \\
\hline$n$ (hyper/norm) & $108 / 618$ & $80 / 807$ & $53 / 305$ & $63 / 415$ & & \\
\hline \multicolumn{7}{|l|}{$\beta_{0}+b_{0}\left(\mathrm{~kg} / \mathrm{m}^{2}\right)$} \\
\hline Hyper & $25.6 \pm 5.3$ & $26.9 \pm 7.5$ & $25.7 \pm 5.9$ & $27.4 \pm 6.6$ & 0.903 & 0.678 \\
\hline Norm & $23.7 \pm 4.3^{* *}$ & $22.7 \pm 4.5$ & $23.5 \pm 4.8^{* *}$ & $25.1 \pm 5.8$ & 0.624 & $<0.001$ \\
\hline$p^{\mathrm{a}}$ & $<0.001$ & $<0.001$ & $<0.001$ & $<0.001$ & & \\
\hline \multicolumn{7}{|l|}{$\beta_{1}+b_{1}\left(\mathrm{~kg} / \mathrm{m}^{2}\right)$} \\
\hline Hyper & $0.55 \pm 0.21$ & $0.63 \pm 0.32$ & $0.61 \pm 0.25$ & $0.70 \pm 0.37$ & 0.164 & 0.187 \\
\hline Norm & $0.47 \pm 0.20 * *$ & $0.40 \pm 0.27$ & $0.49 \pm 0.25^{* *}$ & $0.56 \pm 0.35$ & 0.136 & $<0.001$ \\
\hline$p^{\mathrm{a}}$ & $<0.001$ & $<0.001$ & $<0.001$ & $<0.001$ & & \\
\hline \multicolumn{7}{|l|}{$\beta_{2}+b_{2}\left(\mathrm{~kg} / \mathrm{m}^{2}\right)$} \\
\hline Hyper & $-0.116 \pm 0.115$ & $-0.092 \pm 0.139$ & $-0.103 \pm 0.138$ & $-0.108 \pm 0.142$ & 0.542 & 0.479 \\
\hline Norm & $-0.104 \pm 0.087 * *$ & $-0.083 \pm 0.086$ & $-0.101 \pm 0.088$ & $-0.106 \pm 0.108$ & 0.584 & $<0.001$ \\
\hline$p^{\mathrm{a}}$ & 0.230 & 0.191 & 0.713 & 0.413 & & \\
\hline \multicolumn{7}{|l|}{$\beta_{3}+b_{3}\left(\mathrm{~kg} / \mathrm{m}^{2}\right)$} \\
\hline Hyper & $0.002 \pm 0.009 *$ & $-0.001 \pm 0.009$ & $-0.0003 \pm 0.014$ & $-0.001 \pm 0.010$ & 0.178 & 0.922 \\
\hline Norm & $0.002 \pm 0.007$ & $0.002 \pm 0.008$ & $0.001 \pm 0.011$ & $0.002 \pm 0.010$ & 0.287 & 0.114 \\
\hline$p^{\mathrm{a}}$ & 0.597 & $<0.001$ & 0.038 & 0.094 & & \\
\hline
\end{tabular}

Data are means $\pm \mathrm{SD}$, unless otherwise indicated

${ }^{a} p$ values for difference in $\beta \mathrm{s}+$ bs between hyperglycaemia and normoglycaemia groups, adjusted for average age, with additional adjustment for $\beta_{0}+$ $b_{0}$ for other curve parameters

${ }^{*} p<0.05, * * p<0.01$ for a sex difference within racial groups

Hyper, hyperglycaemia; Norm, normoglycaemia 


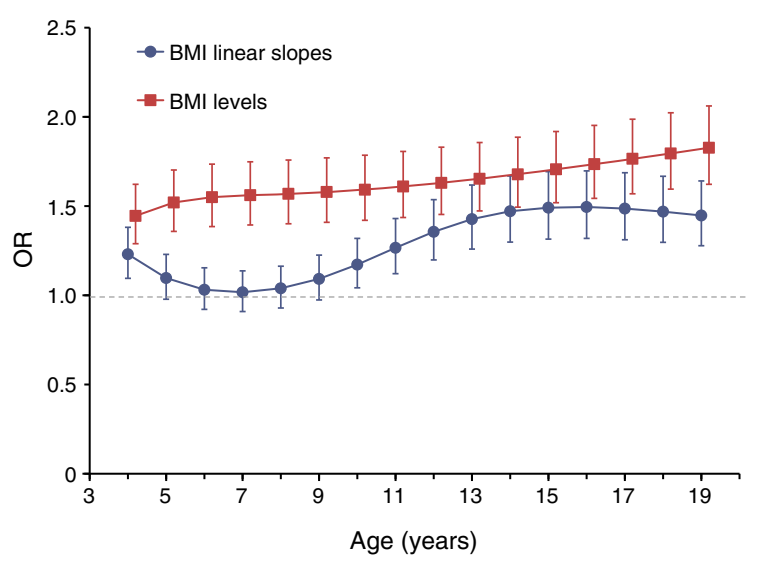

Fig. 3 ORs and 95\% CIs of model-estimated BMI levels and level-adjusted BMI linear slopes for adult hyperglycaemia (prediabetes and type 2 diabetes, $n=304$ ) by childhood/adolescence age windows, adjusted for adult age, sex, race, smoking and alcohol drinking

BMI slopes increased with increasing childhood age. The ORs were not significant during the age period 5-9 years, whereas the ORs were significantly greater than 1.0 during the age period 10-19 years (OR 1.17-1.50), with a peak (OR $1.50, p<0.001)$ at age 16 years. In contrast, the associations between model-estimated BMI levels and adult hyperglycaemia did not show such an age-related trend, although the ORs ranging from 1.45 to 1.83 were all significant at childhood ages 4-19 years after adjustment for confounders. ESM Fig. 2 shows the ORs of levels and level-adjusted linear slopes of childhood BMI for adult hyperglycaemia by race-sex groups. The trend in ORs was similar in white and black males but different in black females, with significant OR values of levels and slopes at age 4 years and non-significant OR values of slopes from 5 to 19 years.

ESM Fig. 3 shows ORs of model-estimated, leveladjusted linear slopes of BMI for adult prediabetes $(n=$ $164)$ and type 2 diabetes $(n=140)$ by childhood age. The OR values were different (type 2 diabetes $>$ prediabetes) before 12 years of age, but prediabetes and type 2 diabetes had similar trends in ORs during childhood. Of interest, the ORs were significantly greater than 1.0 during the adolescence period of 13-19 years for both prediabetes and type 2 diabetes. As shown in ESM Table 4, ORs of modelestimated linear slopes of BMI for hyperglycaemia were significant in early adulthood from 20 to 30 years, ranging from 1.13 to 1.41 , and in mid-adulthood from 44 to 50 years, ranging from 0.63 to 0.83 . ORs of modelestimated levels of BMI for hyperglycaemia were all significant during adulthood, ranging from 1.69 to 2.14, with similar trends in race-sex groups. ORs of model-estimated levels of BMI for type 2 diabetes and prediabetes were all significant during adulthood, ranging from 1.64 to 2.39 and from 1.56 to 1.98 , respectively.

\section{Discussion}

In this community-based longitudinal cohort with repeated measurements of BMI from childhood to midlife, we have demonstrated that level-independent BMI slopes at age 10-19 years were significantly associated with adult hyperglycaemia. Despite the overwhelming evidence regarding the predictive value of childhood BMI levels for later-life diabetes, no previous studies have concurrently considered the importance of linear slopes and levels of BMI at different childhood age points for the prediction of adult diabetes. The observations from this study provide new insights into the early-life origins of diabetes and emphasise the importance of level-independent BMI trajectories during the adolescence period for assessing diabetes risk in later life.

To date, limited information has been available to detail trajectory parameters of longitudinal BMI profiles in childhood and their relationship to the development of diabetes in later life. In the current study, we found that the hyperglycaemic group had higher BMI levels $\left(\beta_{0}+b_{0}\right)$ and linear slopes $\left(\beta_{1}+b_{1}\right)$ at age 20.1 years and a consistently lower rate of increase $\left(\beta_{2}+b_{2}\right)$ around $20-35$ years of age than the normoglycaemic group in all race-sex groups, and significantly smaller values of change in the rate of increase $\left(\beta_{3}+b_{3}\right)$ around 36-51 years of age in white female and black male hyperglycaemic participants than in the normoglycaemic group. The lower values of $\beta_{3}+b_{3}$ among hyperglycaemic participants might be explained by the regression-to-themean phenomenon. The change in the rate of BMI increase tended to slow down in middle-aged hyperglycaemic adults because there were more adults in this group with higher BMI levels. The results of the current study indicate that the increase in BMI during early life is important for the development of type 2 diabetes.

The associations between BMI slopes at different childhood ages and adult hyperglycaemia varied during the preadolescence and adolescence periods. Importantly, these associations were independent of childhood BMI levels at the same age points. Limited literature is available for comparison in this regard. We found that the model-estimated and level-adjusted linear slopes of BMI during the age period 10-19 years were significantly and positively associated with middle-aged adult hyperglycaemia, indicating that the adolescence age period is a crucial window for the development of hyperglycaemia in later life. The sexual maturation process during the adolescence period is characterised by a complex interplay among various gonadal and adrenal steroid hormones, growth hormones and growth factors. These factors change dramatically during this age period [21, 22]. Previous studies, including the Bogalusa Heart Study, have indicated that glucose and insulin levels during adolescence are mediated by these factors, independent of adiposity [23-25]. The findings from this and previous studies suggest that adolescence is a critical temporal window for 
BMI control to reduce the risk of adult hyperglycaemia [11, 13-16, 26-29].

The trends in ORs of level-independent BMI slopes for prediabetes and type 2 diabetes were different. The ORs for type 2 diabetes were significantly greater than 1.0 for all childhood ages, while the ORs for prediabetes were significantly greater than 1.0 only in the adolescence period of 13-19 years. In contrast to the adolescence period, the level-independent BMI slopes during the pre-adolescence period of 6-8 years showed significantly negative associations with adult prediabetes. The underlying mechanisms for the observed differential associations at different growth periods in childhood remained to be dissected and cannot be addressed by this population-based observational study. Childhood growth and development undergo consecutive, programmed periods with hormones that may play a major role. How early-life growth and development programming influences BMI trajectories and then alters adult glucose levels remains to be fully understood. This negative association is an important and intriguing finding that has not been previously reported and warrants further study to confirm in other populations.

The current study has important strengths. Body weight and height were repeatedly measured over time using a consistent study protocol. Participants were examined at least four times, which allowed for reliable parameter estimates in growth-curve modelling. Growth-curve models fit reasonably well with the observed data, which adds weight to the validity of derived rate of change in BMI at different childhood ages. In addition, the associations between rate of change in childhood BMI and adult hyperglycaemia were generally consistent across the four racesex groups. On the other hand, in this study cohort, childhood BMI was measured every 2-3 years at unequal intervals and, therefore, the rate of change in BMI at different ages had to be estimated through statistical modelling. This model-estimation approach needs to be validated by using childhood BMI measured in 1-year intervals to assess diabetes risk in later life.

In summary, our study demonstrates that not only are the absolute values of BMI during childhood associated with adult hyperglycaemia, but also that level-independent BMI slopes predict adult diabetes risk, particularly at ages after 10 years during childhood. The findings support the early prevention of and interventions for childhood obesity. The significantly positive correlations between levels and slopes of BMI at childhood ages found in this study indicate that children who have a steeper increasing slope of BMI, especially during adolescence, have a greater risk of obesity and thus a higher risk of diabetes. These observations suggest that developing prevention strategies for obesity control in adolescence might be effective in reducing the adult diabetes risk burden. Further studies are needed to explore the underlying mechanisms, such as hormonal changes during adolescence, for the observed differential associations between BMI slopes during childhood and adult diabetes.
Acknowledgements The Bogalusa Heart Study is a joint effort of many investigators and staff members whose contributions are gratefully acknowledged.

Data availability All data and materials are publicly available at the NHLBI Biologic Specimen and Data Repository and can be accessed at https://biolincc.nhlbi.nih.gov/studies/bhs.

Funding This study was supported by grants 81673271 and 81701141 from the National Natural Science Foundation of China, R01HL121230 from the NHLBI, R03AG060619 from the National Institute on Aging and P20GM109036 from the National Institute of General Medical Sciences of the National Institutes of Health.

Duality of interest The authors declare that there is no duality of interest associated with this manuscript.

Author contributions TZ, JX and WC contributed to conception and design, acquisition of data, analysis and interpretation of data and drafting of the article, and gave final approval of the version to be published. SL contributed to analysis and interpretation of data and revising the article critically and gave final approval of the version to be published. $\mathrm{LAB}, \mathrm{JH}$ and $\mathrm{PKW}$ contributed to supervision of collecting and analysing the data, interpretation of data and revising the article and gave final approval of the version to be published. WC is responsible for the integrity of the work as a whole.

\section{References}

1. Wei GS, Coady SA, Reis JP et al (2015) Duration and degree of weight gain and incident diabetes in younger versus middle-aged black and white adults: ARIC, CARDIA, and the Framingham Heart Study. Diabetes Care 38(11):2042-2049. https://doi.org/10. 2337/dc14-2770

2. Hamman RF, Wing RR, Edelstein SL et al (2006) Effect of weight loss with lifestyle intervention on risk of diabetes. Diabetes Care 29(9):2102-2107. https://doi.org/10.2337/dc06-0560

3. Hsia DS, Larrivee S, Cefalu WT, Johnson WD (2015) Impact of lowering BMI cut points as recommended in the revised American Diabetes Association's Standards of Medical Care in Diabetes-2015 on diabetes screening in Asian Americans. Diabetes Care 38(11): 2166-2168. https://doi.org/10.2337/dc15-0299

4. Lotta LA, Abbasi A, Sharp SJ et al (2015) Definitions of metabolic health and risk of future type 2 diabetes in BMI categories: a systematic review and network meta-analysis. Diabetes Care 38(11): 2177-2187. https://doi.org/10.2337/dc15-1218

5. Nguyen QM, Srinivasan SR, Xu J-H et al (2008) Changes in risk variables of metabolic syndrome since childhood in pre-diabetic and type 2 diabetic subjects: the Bogalusa Heart Study. Diabetes Care 31(10):2044-2049. https://doi.org/10.2337/dc08-0898

6. Zhang T, Zhang H, Li Y et al (2017) Long-term impact of temporal sequence from childhood obesity to hyperinsulinemia on adult metabolic syndrome and diabetes: the Bogalusa Heart Study. Sci Rep 7(1):43422. https://doi.org/10.1038/srep43422

7. Koskinen J, Magnussen CG, Sinaiko A et al (2017) Childhood age and associations between childhood metabolic syndrome and adult risk for metabolic syndrome, type 2 diabetes mellitus and carotid intima media thickness: the International Childhood Cardiovascular Cohort Consortium. J Am Heart Assoc 6:e005632

8. Hannon TS, Rao G, Arslanian SA (2005) Childhood obesity and type 2 diabetes mellitus. Pediatrics 116(2):473-480. https://doi.org/ 10.1542/peds.2004-2536 
9. Srinivasan S, Myers L, Berenson G (2002) Predictability of childhood adiposity and insulin for developing insulin resistance syndrome (syndrome $\mathrm{X}$ ) in young adulthood the Bogalusa Heart Study. Diabetes 51(1):204-209. https://doi.org/10.2337/diabetes.51.1.204

10. Geng T, Smith CE, Li C, Huang T (2018) Childhood BMI and adult type 2 diabetes, coronary artery diseases, chronic kidney disease, and cardiometabolic traits: a Mendelian randomization analysis. Diabetes Care 41(5):1089-1096. https://doi.org/10.2337/dc172141

11. Power C, Thomas C (2011) Changes in BMI, duration of overweight and obesity, and glucose metabolism: 45 years of followup of a birth cohort. Diabetes Care 34(9):1986-1991. https://doi. org $/ 10.2337 / \mathrm{dc} 10-1482$

12. Dwyer T, Sun C, Magnussen CG et al (2013) Cohort profile: the international childhood cardiovascular cohort (i3C) consortium. Int J Epidemiol 42(1):86-96. https://doi.org/10.1093/ije/dys004

13. Tirosh A, Shai I, Afek A et al (2011) Adolescent BMI trajectory and risk of diabetes versus coronary disease. N Engl J Med 364(14): 1315-1325. https://doi.org/10.1056/NEJMoa1006992

14. Chiu CJ, Wray LA, Lu FH, Beverly EA (2013) BMI change patterns and disability development of middle-aged adults with diabetes: a dual trajectory modeling approach. J Gen Intern Med 28(9): 1150-1156. https://doi.org/10.1007/s11606-013-2399-z

15. Lammi N, Moltchanova E, Blomstedt PA et al (2009) Childhood BMI trajectories and the risk of developing young adult-onset diabetes. Diabetologia 52(3):408-414. https://doi.org/10.1007/ s00125-008-1244-0

16. Eriksson JG, Kajantie E, Lampl M, Osmond C (2015) Trajectories of body mass index amongst children who develop type 2 diabetes as adults. J Intern Med 278(2):219-226. https://doi.org/10.1111/ joim. 12354

17. Berenson GS, McMahan CA, Voors AW, Webber LS, Srinivasan SR (1980) Cardiovascular risk factors in children: the early natural history of atherosclerosis and essential hypertension. Oxford University Press, New York

18. American Diabetes Association (2010) Diagnosis and classification of diabetes mellitus. Diabetes Care 33(Suppl 1):S62-S69. https:// doi.org/10.2337/dc10-S062

19. Cook NR, Rosner BA, Chen W et al (2004) Using the area under the curve to reduce measurement error in predicting young adult blood pressure from childhood measures. Stat Med 23(22):3421-3435. https://doi.org/10.1002/sim.1921

20. Chen W, Li S, Srinivasan SR et al (2005) Autosomal genome scan for loci linked to blood pressure levels and trends since childhood: the Bogalusa Heart Study. Hypertension 45(5):954-959. https://doi. org/10.1161/01.HYP.0000161881.02361.11

21. Lee PA, Xenakis T, Winer J, Matsenbaugh S (1976) Puberty in girls: correlation of serum levels of gonadotropins, prolactin, androgens, estrogens, and progestins with physical changes. J Clin Endocrinol Metab 43(4):775-784. https://doi.org/10.1210/jcem43-4-775

22. Lee PA, Migeon CJ (1975) Puberty in boys: correlation of plasma levels of gonadotropins, androgens (testosterone, androstenedione, dehydroepiandrosterone and its sulfate), estrogens (estrone and estradiol) and progestins (progesterone and 17-hydroxyprogesterone). J Clin Endocrinol Metab 41(3):556-562. https://doi.org/10.1210/ jcem-41-3-556

23. Gordon CM, Binello E, LeBoff MS et al (2006) Relationship between insulin-like growth factor I, dehydroepiandrosterone sulfate and proresorptive cytokines and bone density in cystic fibrosis. Osteoporos Int 17(5):783-790. https://doi.org/10.1007/s00198005-0058-x

24. Srinivasan SR, Freedman DS, Sundaram GS et al (1986) Racial (black-white) comparisons of the relationship of levels of endogenous sex hormones to serum lipoproteins during male adolescence: the Bogalusa Heart Study. Circulation 74(6):1226-1234. https:// doi.org/10.1161/01.CIR.74.6.1226

25. Jiang X, Srinivasan SR, Dalferes ER, Berenson GS (1997) Plasma insulin-like growth factor 1 distribution and its relation to blood pressure in adolescents: the Bogalusa Heart Study. Am J Hypertens 10(7):714-719. https://doi.org/10.1016/S08957061(97)00065-4

26. Mano Y, Yokomichi H, Suzuki K et al (2015) Do body mass index trajectories affect the risk of type 2 diabetes? A case-control study. BMC Public Health 15(1):718. https://doi.org/10.1186/s12889015-2073-y

27. Kuwahara K, Honda T, Nakagawa T et al (2017) Body mass index trajectory patterns and changes in visceral fat and glucose metabolism before the onset of type 2 diabetes. Sci Rep 7(1):43521. https:// doi.org/10.1038/srep43521

28. Heianza Y, Arase Y, Kodama S et al (2015) Trajectory of body mass index before the development of type 2 diabetes in Japanese men: Toranomon Hospital Health Management Center Study 15. J Diabetes Investig 6(3):289-294. https://doi.org/10.1111/jdi.12308

29. Chiu CJ, Li SL, Wu C-H, Du YF (2016) BMI trajectories as a harbinger of pre-diabetes or underdiagnosed diabetes: an 18-year retrospective cohort study in Taiwan. J Gen Intern Med 31(10): 1156-1163. https://doi.org/10.1007/s11606-016-3750-y 